\title{
Overlapping volumes in re-irradiation for head and neck cancer - an important factor for patient selection
}

\author{
Anna Embring ${ }^{1,2^{*}}$ D, Eva Onjukka ${ }^{2,3}$, Claes Mercke ${ }^{1,2}$, Ingmar Lax $^{3}$, Anders Berglund $^{4}$, Sara Bornedal ${ }^{3}$, \\ Berit Wennberg ${ }^{3}$ and Signe Friesland ${ }^{1,2}$
}

\begin{abstract}
Background: There is a lack of consensus concerning the definition of re-irradiation and re-irradiation volumes in head and neck cancer (HNC). The aim of the present study is to introduce a more strict definition of the reirradiated volume that might better predict the risk of serious side-effects from treatment.

Methods: Fifty-four consecutive patients re-irradiated for HNC cancer were retrospectively analysed. CT images were deformably registered and the dose distributions accumulated after conversion to EQD2. Patients with a cumulative dose of $\geq 100 \mathrm{~Gy}$ in the overlapping volume (V100) were included in the study. Survival data and radiation-related acute and late toxicities were recorded.

Results: The overall survival of all included patients at 2 and 5 years was 42.6 and $27.3 \%$ respectively and the progression free survival at 2 and 5 years was 32.5 and $28.5 \%$ respectively. The overall rate of any event of severe (grade $\geq 3$ ) acute and late toxicity was 26 and 51\%, respectively. We found that severe acute toxicity was more common in patients who had a larger overlapping volume (V100 > mean) where $43 \%$ of the patients experienced grade $\geq 3$ acute toxicity, compared to the patients with smaller overlapping volumes ( $100<$ mean) where only $11 \%$ had severe toxicity $(p=0.02)$. The seemingly high rates of late toxicity in the present study could be due to the use of a more strict definition of re-irradiation. In previous studies also patients with low dose overlap are included and our results imply that there is a risk that previous studies might have overestimated the risk-benefit ratio in re-irradiation of HNC.

Conclusions: Our study describes the outcome of a patient material where a more strict definition of the reirradiated volume is used. With this definition, which could better describe the volume of highest risk for serious complications, we found that larger such overlapping volumes result in an increase in severe acute side-effects. A clear definition of re-irradiation and re-irradiation volumes is of utmost importance for future studies of HNC to make results from different studies comparable.
\end{abstract}

Keywords: Re-irradiation, Head and neck cancer, Re-irradiation volume, Cumulative dose, Overlap, HNSCC

\footnotetext{
* Correspondence: anna.embring@ki.se

'Department of Oncology, Karolinska University Hospital, Anna Steckséns

gata 41, 17176 Stockholm, Solna, Sweden

${ }^{2}$ Department of Oncology-Pathology, Karolinska Institute, Stockholm, Sweden

Full list of author information is available at the end of the article
}

(C) The Author(s). 2020 Open Access This article is licensed under a Creative Commons Attribution 4.0 International License, which permits use, sharing, adaptation, distribution and reproduction in any medium or format, as long as you give appropriate credit to the original author(s) and the source, provide a link to the Creative Commons licence, and indicate if changes were made. The images or other third party material in this article are included in the article's Creative Commons. licence, unless indicated otherwise in a credit line to the material. If material is not included in the article's Creative Commons licence and your intended use is not permitted by statutory regulation or exceeds the permitted use, you will need to obtain permission directly from the copyright holder. To view a copy of this licence, visit http://creativecommons.org/licenses/by/4.0/ The Creative Commons Public Domain Dedication waiver (http://creativecommons.org/publicdomain/zero/1.0/) applies to the data made available in this article, unless otherwise stated in a credit line to the data. 


\section{Background}

Local recurrence is the predominant pattern of failure after treatment of advanced head and neck cancer (HNC) [1]. A recurrence often occurs in an area already treated with radiotherapy, which needs to be taken into account when considering different treatment options. The treatment method of choice is surgical resection [2], but for many patients, surgery is not possible due to unresectable tumour or co-morbidity. These patients may be offered palliative chemotherapy, associated with a median progression-free survival (PFS) of 3-4 months and a median overall survival (OS) of 6-10 months [3-5]. In recent years, immunotherapy has also emerged as a treatment option for patients with recurrent HNC. Immunotherapy can offer long-lasting remission for some patients, without the toxicity of chemotherapy, but only a limited group of patients with recurrent $\mathrm{HNC}$ benefit from immunotherapy and the knowledge regarding predictive factors in this group is still limited $[6,7]$.

Historically re-irradiation was avoided due to the risk of severe toxicity. However, several studies have now shown that re-irradiation for $\mathrm{HNC}$ is a treatment option for selected patients and can offer long-term disease control, or even cure [8-12]. In a multi-institution study including 412 patients with $\mathrm{HNC}$, Ward et al. demonstrated an actuarial OS rate of $40 \%$ at 2 years [8], and in a recently published single-institution study by Rühle et al. the OS at 2 and 5 years was 52.3 and 34.3\%, respectively, after re-irradiation for HNC [13]. However, re-irradiation for $\mathrm{HNC}$ still remains challenging mainly because of the concern of toxicity, and it would be useful to find predictive factors to guide patient selection. Deriving a consensus on patient selection from the literature is difficult due to varying or vague definitions of reirradiation. Many studies suggest no definition of reirradiation other than repeat radiotherapy in the head and neck area and it remains to be established what retreatment parameters have clinical significance. It would be of great value if there was a consensus on how to define re-irradiation and re-irradiation volumes, as this would facilitate comparisons between different studies and in turn provide more solid data for patient selection. It is even possible that the value of re-irradiation in HNC has been overestimated due to the vague definition of re-irradiation in past studies, allowing the inclusion of patients who in fact had no overlapping volumes of high dose.

Intensity modulated radiotherapy (IMRT) has been shown to improve local control and survival in the treatment of head and neck cancer [14-17]. That more conformal techniques result in better patient outcome supports the hypothesis that the size of the irradiated volume might have an impact on patient outcome. It may be assumed that a relevant volume is the overlapping volume, i.e., the volume that is irradiated both at the initial treatment and then again at reirradiation. This differs from the re-treatment volume, which is the volume treated at the time of re-irradiation, i.e., the planning target volume (PTV). However, as the latter is easier to obtain, this is the parameter typically studied in the literature, and it has been shown that the size of the PTV at re-treatment affects patient outcome $[18,19]$. However, a large re-treatment volume might just represent a large tumour burden at re-irradiation and not necessarily a large overlapping volume. Today when treatment-planning data from the primary treatment most often is available electronically, it seems reasonable to move on from using the PTV at re-treatment as a surrogate for evaluating the re-irradiation volume. Therefore, the current study explores the impact on patient outcome from the overlapping volume rather than the re-treatment volume. Dosimetric data from both the primary treatment and the re-irradiation were gathered, making it possible to determine the overlapping volume.

In this work we propose a definition of the reirradiated volume which better represents both treatments. The treatment outcome after re-irradiation for HNC in our institution, as per the proposed definition, is also evaluated in relation to patient- and treatment characteristics.

\section{Material and methods \\ Patients}

Fifty-four consecutive patients re-irradiated for HNC between 2011 and 2017 in our institution were retrospectively analysed. The inclusion criteria were initial radiotherapy with curative intent ( $\geq 60 \mathrm{~Gy}$ ) for $\mathrm{HNC}$, a second course of radiotherapy for recurrence of $\mathrm{HNC}$ or second primary $\mathrm{HNC}$ where the intent was to achieve cure or local control ( $\geq 40 \mathrm{~Gy}$ ), and an overlapping volume of these two treatments (Fig. 1). Consistent with this definition of re-irradiation, the cumulative dose in what was considered the overlapping volume was $\geq 100$ Gy in EQD2, i.e. V100. To account for different fractionation schedules and heterogeneous dose distributions, all radiotherapy doses are reported in an equivalent dose of 2 Gy fractions (EQD2) based on the linear-quadratic model [20], using $\alpha / \beta=3$ Gy.

A re-treatment dose $\geq 40$ Gy was considered to reflect the clinician's intent to achieve local control, in contrast to palliation; the same dose has been used in other studies of re-irradiation as a cut-off for significant reirradiation dose $[8,18]$. Thus, patients re-irradiated with palliative doses were excluded, as were patients reirradiated for other tumours than HNC. One patient was also excluded because of missing data from the initial radiotherapy treatment. Three patients went on to have a second course of re-irradiation. Two of these patients 


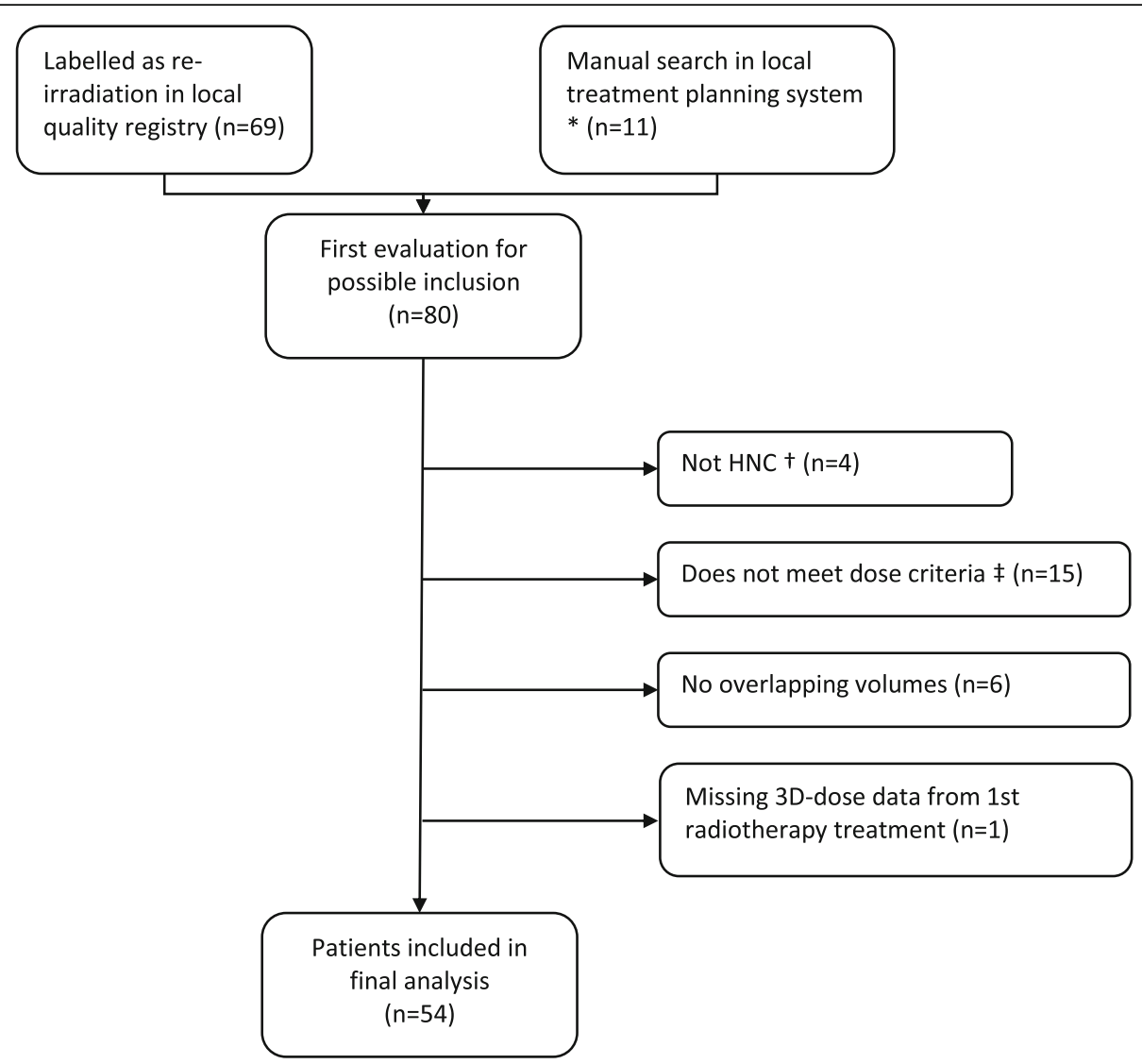

Fig. 1 Flow chart of included patients. Abbreviations: HNC - Head and neck cancer. * All patients with a HNC diagnosis, who had at least 2 courses of radiotherapy and at least 1 course of radiotherapy with curative intent, were reviewed in the treatment planning system for inclusion, in order to find relevant patients who had not been recorded in the quality registry. +1 malignant melanoma, 1 CNS tumour, 2 Merkel cell carcinoma (face). ₹ palliative radiotherapy

had a second recurrence and one patient had a new second primary HNC. For these three patients the overlapping volumes and near-maximum doses were extracted from the total cumulative radiotherapy given. Patient and treatment characteristics at the initial treatment are reported in Table 1.

In our institution approximately 230 patients with primary HNC are treated with curative intent every year. At first presentation patients are typically treated with chemoradiotherapy. Oropharyngeal cancer will typically receive definitive chemoradiotherapy without prior surgery and tumours of the oral cavity will typically undergo surgery before radiotherapy. During the studied period, patients with a tumour in the base of tongue often received a brachytherapy boost to the primary tumour after completing external beam radiotherapy.

The study was approved by the National Ethical Review Authority.

\section{Treatment}

At re-irradiation, $93 \%$ of the patients were treated with highly conformal radiotherapy: either with IMRT or volumetric modulated arc therapy (VMAT), brachytherapy or a combination of external-beam radiotherapy and brachytherapy (Table 2). In the original treatments multiple techniques and modalities were used, including brachytherapy and external-beam therapy with 6 and 18 MV photon fields, and sometimes electrons. All treatment plans were computed tomography $(\mathrm{CT})$ based and for all except four patients, both the original treatment plan and the re-treatment plan were available electronically. For the patients where the original treatment plan was not available electronically, plans were reconstructed on the re-treatment planning-CT from original treatment data available as print-outs (including field parameters, beam apertures, a selection of CT slices with isodoses and dose-volume histograms), to get complete dose data for all the included patients and all treatment courses. The reconstructions were performed by an experienced physicist; field shapes and weights were adapted to the anatomy in the available CT images, resulting in a plan reflecting the clinical practice at the time of treatment. Treatments were originally planned in Eclipse (Varian, USA), TMS (Helax, Sweden) or 
Table 1 Patient and treatment characteristics at first presentation. Tumour stage according to American Joint Committee on Cancer (AJCC) Cancer Staging Manual 7th edition

\begin{tabular}{|c|c|c|}
\hline $\begin{array}{l}\text { Patient and treatment characteristics } \\
\text { at first presentation }\end{array}$ & Number & $\overline{\text { Percent (range) }}$ \\
\hline Male & 35 & 65 \\
\hline Female & 19 & 35 \\
\hline Median age (years) & 59 & $(33-81)$ \\
\hline \multicolumn{3}{|l|}{ Tumor site } \\
\hline Larynx & 6 & 11 \\
\hline Oropharyngeal $^{a}$ & 18 & 33 \\
\hline Nasopharyngeal & 1 & 2 \\
\hline Hypopharyngeal & 5 & 9 \\
\hline Oral cavity & 17 & 31 \\
\hline Unknown primary & 2 & 4 \\
\hline Sino/nasal & 3 & 6 \\
\hline Salivary gland & 2 & 4 \\
\hline \multicolumn{3}{|l|}{ Histology } \\
\hline Squamous cell carcinoma & 46 & 85 \\
\hline Adenoid cystic carcinoma & 5 & 9 \\
\hline Other ${ }^{b}$ & 3 & 6 \\
\hline \multicolumn{3}{|l|}{ Tumor Stage } \\
\hline । & 5 & 9 \\
\hline$\|$ & 11 & 20 \\
\hline III & 7 & 13 \\
\hline IVa & 28 & 52 \\
\hline $\mathrm{IVb}$ & 3 & 6 \\
\hline \multicolumn{3}{|l|}{ T-stage } \\
\hline 0 & 2 & 4 \\
\hline 1 & 9 & 17 \\
\hline 2 & 22 & 41 \\
\hline 3 & 4 & 7 \\
\hline 4 & 17 & 31 \\
\hline Median treatment dose (Gy) & 68 & $(60-79)$ \\
\hline
\end{tabular}

${ }^{a}$ Association with human papilloma virus (HPV): 11 HPV-positive, 2 HPVnegative, $5 \mathrm{HPV}$ status unknown

$\mathrm{b}_{1}$ salivary duct cancer, 1 undifferentiated non-keratinizing cancer, 1 adenocarcinoma

Pinnacle (Philips Radiation Oncology Systems, USA) and any reconstructions were made in Eclipse. All externalbeam dose distributions were calculated in Eclipse using the AAA algorithm, also for plans imported from a different system. Brachytherapy treatments were planned in Oncentra (Elekta, Sweden).

The 3D-dose distributions of all included externalbeam plans and brachytherapy plans were exported to an in-house application converting the dose in each voxel to EQD2. The CT-, structure- and dose data were imported into a research version of Raystation (Raysearch Laboratories, Sweden) where, for each patient, the planning-CT images were registered to the most recent CT used for external-beam planning, using a greylevel based non-rigid registration. The deformed dosedistribution from each treatment was calculated on the reference $\mathrm{CT}$, finally giving the cumulative dose distribution in EQD2 including all the treatment plans. The dose to the hottest $1 \mathrm{~cm}^{3}$ (D1cc) of the patient volume, i.e. the near-maximum dose, as well as V100, were extracted from the cumulative dose distribution.

In this study, the accumulated dose from the original treatment and the re-treatment was derived by registering the planning-CT images non-rigidly and summing the deformed 3D dose. Non-rigid image registration has been shown to significantly improve the estimation of accumulated dose for re-irradiation [21]. While image registration in the head-and-neck region is challenging due to anatomical differences naturally appearing over time and due to different tilts of the head and different mouth fixation used for different treatment courses and modalities, non-rigid registration techniques appear to perform well for this anatomical site [22]. However, the CT images used for brachytherapy planning had extensive artefacts and a very limited field-of-view. For this reason, the brachytherapy planning-CT was never used as a reference image. Despite the challenges, visual evaluation of each non-rigid registration showed a good result.

\section{Oncologic and toxicity outcomes}

Data of patient outcome were collected from a local quality registry, with prospectively gathered data, and supplemented with a review of medical records. Acute and late toxicities were graded according to Radiation Oncology Group (RTOG) and the European Organization for Research and Treatment of Cancer (EORTC) Radiation Morbidity Schema. Patients are invited for routine follow-up visits every 3 months the first 2 years after treatment, and then every 6 months for another 3 years. Patient outcome data were collected and recorded in the local quality registry every 6 months during this time. Toxicities were considered acute if presented within 90 days of the last day of re-irradiation. Any toxicities presenting later were considered late toxicities. Toxicities specifically investigated were mucositis, osteoradionecrosis, soft tissue necrosis, trismus, dysphagia and carotid blowout. The Eastern Cooperative Oncology Group (ECOG) Scale of Performance status (PS) was used to quantify the functional status of the patients.

Oncologic endpoints included OS and PFS. OS was defined as the time between the last day of radiotherapy to the time of death or the last date of clinical follow-up. 
PFS was defined as the time from the last day of reirradiation to the time of progression, death or the last date of clinical follow-up. Progression was defined as either progression on diagnostic imaging, a positive biopsy or a clinical progression assessed by a clinician. Carotid blowout syndrome was defined as massive pharyngeal bleeding in the absence of local recurrence.

\section{Statistics}

The Kaplan-Meier method was used to estimate OS and PFS from the last day of re-irradiation. Re-irradiation dose, overlapping re-treated volume, site of recurrence, PS at re-irradiation, size of PTV, interval between irradiations, definitive versus postoperative re-irradiation, recurrence versus second primary tumour and age were used as predictive variables. The chi-square test was used to test differences in toxicity. A test result below $5 \%$ was considered as statistically significant, and $R$ version 3.6.1 was used for the data management and the analysis.

\section{Results}

Patient and treatment characteristics

A total of 54 patients were included in the analysis. Sixty-nine percent of the patients were treated for recurrent $\mathrm{HNC}$ and $31 \%$ for second primary HNC. The median time from first radiation treatment to re-irradiation was 36 months (range 5.2-177). The median follow-up time after re-irradiation was 20.1 months (range 0-69.9) in all patients and 54.1 months (range 34.3-66.3) in surviving patients. At closure of the database (December 20th, 2018) 11 patients were alive without disease and 2 patients were alive with disease. Causes of death are reported in Table 3.

Median initial treatment dose was 68 Gy (range 6079) and median re-irradiation dose was 59 Gy (range 40-71). For the external beam treatment, the median daily fractionation dose was $2 \mathrm{~Gy}$ at both primary treatment and re-irradiation. Nine patients had an additional brachy boost at the initial treatment and 5 patients had brachytherapy at re-irradiation. Median overlapping volume (V100) was $90 \mathrm{~cm}^{3}$ and median PTV at reirradiation was $145 \mathrm{~cm}^{3}$ (Fig. 2). Patient and treatment characteristics are reported in Tables 1 and 2.

\section{Disease control and overall survival}

The OS of all included patients at 2 and 5 years was 42.6 and $27.3 \%$ respectively and PFS at 2 and 5 years was 32.5 and $28.5 \%$ respectively. Three patients had progressive disease during treatment and were not eligible for PFS evaluation. Patients with a PS of 0 had a greater $(p=$ 0.03 ) OS ( 53.3 and $39.5 \%$, respectively, at 2 and 5 years) compared to patients with PS 1-3 (29.2 and $12.5 \%$, respectively, at 2 and 5 years) (Fig. 3). Patients that were
Table 2 Patient and tumour characteristics at re-irradiation. Abbreviations: VMAT - Volumetric modulated arc therapy, IMRT Intensity modulated radiotherapy, PTV - planning target volume

\begin{tabular}{lll}
\hline $\begin{array}{l}\text { Patient and treatment characteristics } \\
\text { at re-irradiation }\end{array}$ & Number & $\begin{array}{l}\text { Percent } \\
\text { (range) }\end{array}$ \\
\hline $\begin{array}{l}\text { Median age at end of re-irradiation } \\
\text { (years) }\end{array}$ & 63 & $(40-89)$ \\
$\begin{array}{l}\text { Median time between radiations } \\
\text { (months) }\end{array}$ & 36 & $(5-177)$
\end{tabular}

\section{Performance status}

$\begin{array}{lll}0 & 30 & 56 \\ 1 & 21 & 39 \\ 2 & 2 & 4 \\ 3 & 1 & 2\end{array}$

Tumour

Local recurrence $\quad 37 \quad 69$

Secondary primary tumour $\quad 17 \quad 31$

Surgery before re-irradiation

$\begin{array}{lll}\text { No } & 32 & 59 \\ \text { Primary tumour } & 11 & 20 \\ \text { Neck dissection } & 8 & 15 \\ \text { Primary tumour and neck dissection } & 3 & 6\end{array}$

Systemic medical treatment

$\begin{array}{lll}\text { No } & 41 & 76\end{array}$

Induction chemotherapy $\quad 11 \quad 20$

Concurrent chemotherapy (cisplatin) $\quad 1 \quad 2$

Concurrent cetuximab $\quad 1 \quad 2$

Radiotherapy technique at re-irradiation

VMAT/IMRT 45

VMAT/IMRT + brachytherapy $\quad 3 \quad 6$

3D conformal $\quad 4 \quad 7$

3D conformal + brachytherapy $\quad 1 \quad 2$

Brachytherapy

Median re-irradiation dose (Gy)

Median cumulative near max dose,

D1cc (Gy)

Median PTV at re-irradiation $\left(\mathrm{cm}^{3}\right)$

56

39

.

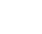

\begin{tabular}{lll} 
Median re-treated volume, $\mathbf{V} 100\left(\mathbf{c m}^{\mathbf{3}}\right)$ & 90 & $(13-668)$ \\
\hline
\end{tabular}

treated with postoperative re-irradiation had both better $(p<0.01)$ OS $(63.6$ and 39.7\%, respectively, at 2 and 5 years) and better ( $p=0.03)$ PFS ( 47.0 and $41.8 \%$, respectively, at 2 and 5 years), compared to patients that were treated with definitive re-irradiation (OS at 28.1 and $18.8 \%$, respectively, at 2 and 5 years and PFS at 22.6 and $19.4 \%$, respectively, at 2 and 5 years) (Fig. 3). This study did not show a significant difference in OS or PFS related to the overlapping volume, PTV-size at reirradiation, interval between irradiations, age at re- 
Table 3 Cause of death

\begin{tabular}{ll}
\hline Cause of death & Number \\
\hline Locoregional disease & 27 \\
Distant metastases & 7 \\
Other disease & 4 \\
Treatment complications & 3 \\
Total & 41 \\
\hline
\end{tabular}

irradiation, D1cc or dose at re-irradiation ( $\geq 55$ Gy vs < 55 Gy $($ EQD2 $\alpha / \beta=10))$.

\section{Toxicity}

The overall rate of any event of severe (grade $\geq 3$ ) acute and late toxicity was 26 and 51\%, respectively. Three patients $(5.6 \%)$ were thought to have died due to treatment-related toxicity. One patient died of acute radiation toxicity and 2 patients died of carotid blowout. The carotid blowouts presented 15 and 38 months after completion of re-irradiation. Severe acute toxicity was more common in patients who had a larger overlapping volume (V100 > mean) where $43 \%$ of the patients experienced grade $\geq 3$ acute toxicity, compared to the patients with smaller overlapping volumes (V100<mean) where only $11 \%$ had severe toxicity $(p=0.02)$ (Table 4$)$. The majority of the cases of severe acute toxicities were mucositis (56\%), followed by trismus (31\%) and other (13\%). We found no significant difference in severe acute toxicity related to the time interval between irradiations. No significant differences could be seen in late severe toxicity in correlation to the overlapping volume, nor could any significant differences be seen in the rate of acute or late severe toxicity in relation to D1cc or size of PTV at re-irradiation.

\section{Discussion}

In our group of patients treated with re-irradiation for HNC the OS at 2 and 5 years was 42.6 and 27.3\%, respectively. This is in accordance with other published data $[8,10]$, even though the definition of re-irradiation differs between studies. Our results show that patients with a good performance status (PS 0) have a significantly higher OS than patients with a worse performance status (PS 1-3). This is also in accordance with previously published literature. For example, in the study by Takiar et al., a multivariate analysis showed that PS > 1 was associated with unfavourable OS [23]. In our study, patients treated with re-irradiation postoperatively had a significantly better OS and PFS than patients treated with definitive re-irradiation. These results are also is in line with already published data $[8,24]$.

The rates of grade $\geq 3$ toxicity were 26 and $51 \%$, for acute and late toxicity respectively. The late side-effect rate is in the higher range of what is previously published. In a review article by Dionisi et al. including 3766 patients from 39 different re-irradiation studies in $\mathrm{HNC}$, the pooled acute and late toxicity rates grade $\geq 3$ were 32 and $29.3 \%$, respectively [25]. One contributing factor to the high rates of late toxicity in the current study could be the relatively long follow-up. It is reasonable to assume that a longer follow-up time will detect more late toxicity and could be a reason for the lower toxicity rate in studies with shorter follow-up time $[8,18]$ compared to the current study. However, another contributing factor to the seemingly high rates of late toxicity could be our strict definition of re-irradiation. Patients with overlapping volumes in the low-dose area only are not included in our cohort, but only patients treated with $\geq 60$ Gy at primary treatment and an overlapping dose of

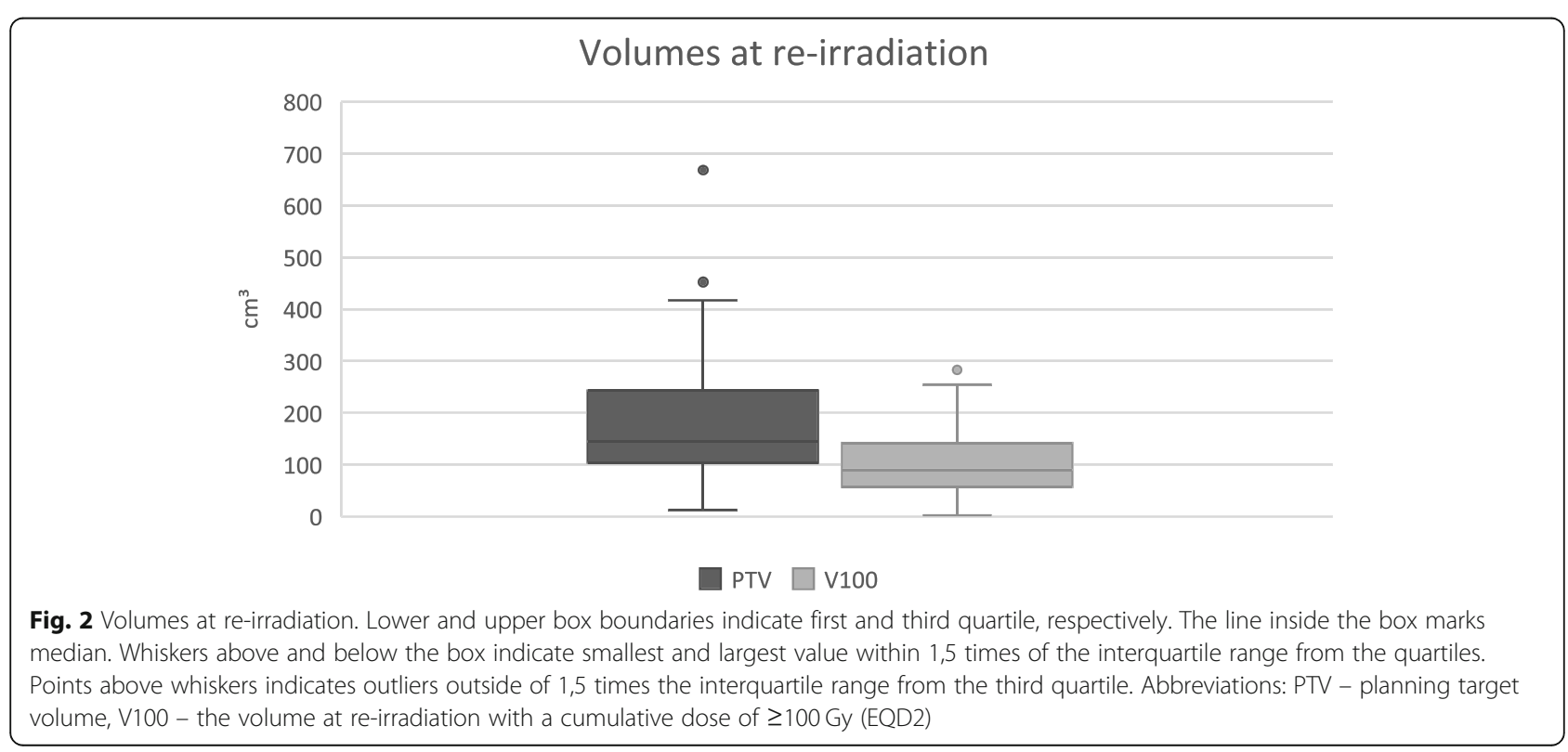




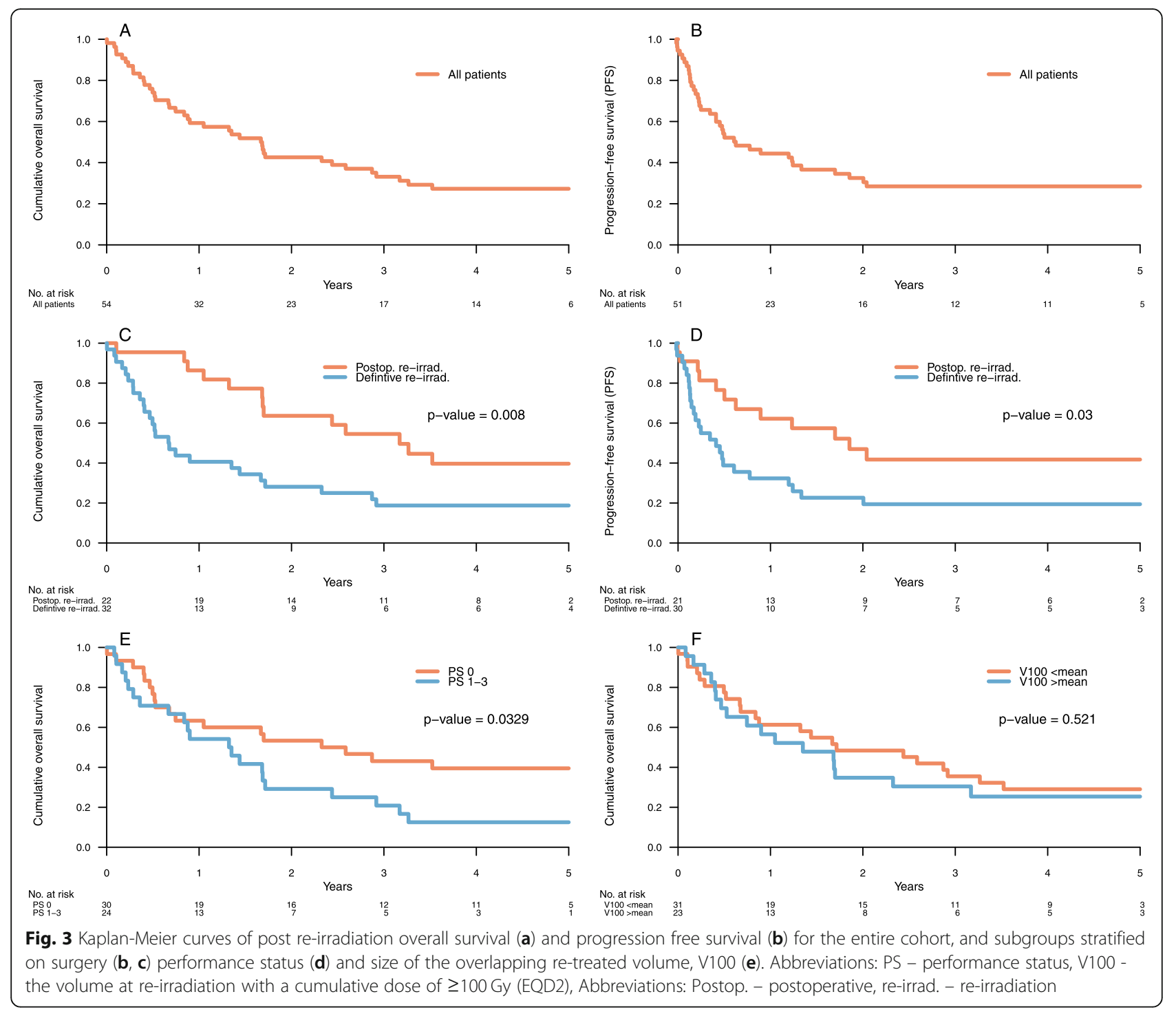

$\geq 40$ Gy at re-irradiation. Previous studies have included also patients with overlapping volumes of lower doses. One could assume that these studies would detect less severe toxicity and lower rates of severe toxicity in the studied populations. Thus, our findings imply that late side effects in re-irradiated HNC patients is a greater problem than previous studies have suggested. Grade 5 toxicity was in level with other published data [10].

Several other groups have shown that re-irradiation is a treatment option for selected patients with recurrent or secondary primary HNC [8-10], but selecting patients for re-irradiation is challenging. Some prognostic factors

Table 4 Side effects after re-irradiation and the correlation to the overlapping volume. Abbreviations: V100 - the volume at reirradiation with a cumulative dose of $\geq 100$ Gy (EQD2). Toxicity was graded according to Radiation Oncology Group (RTOG) and the European Organization for Research and Treatment of Cancer (EORTC) Radiation Morbidity Schema

\begin{tabular}{|c|c|c|c|c|c|c|c|c|}
\hline \multirow{3}{*}{$\begin{array}{l}\text { Side effects } \\
\text { after re-irradiation }^{a}\end{array}$} & \multicolumn{4}{|c|}{ Acute } & \multicolumn{4}{|c|}{ Late } \\
\hline & \multicolumn{2}{|c|}{ V100 < mean } & \multicolumn{2}{|c|}{ V100 > mean } & \multicolumn{2}{|c|}{ V100 < mean } & \multicolumn{2}{|c|}{ V100 > mean } \\
\hline & $\mathrm{n}$ & $\%$ & $n$ & $\%$ & $\mathrm{n}$ & $\%$ & $\mathrm{n}$ & $\%$ \\
\hline Grade $0-2$ & 24 & 89 & 13 & 57 & 13 & 59 & 5 & 33 \\
\hline Grade $\geq 3$ & 3 & 11 & 10 & 43 & 9 & 41 & 10 & 67 \\
\hline
\end{tabular}


have been identified: patients with a longer time interval between primary treatment and re-irradiation have better prognosis $[24,26]$, the absence of organ dysfunction (feeding tube or tracheostomy dependence) is favourable $[8,27]$, and higher doses at re-irradiation yield better outcomes $[14,24]$. There are also various tools available to help guide clinicians in their decision making. Tanvetyanon et al. suggested a nomogram for predicting 24month survival probability [27], and Ward et al. have constructed a nomogram for predicting severe late toxicity at two years after re-irradiation [28]. In the latter study it was suggested that late toxicity may be more dependent on patient- and disease factors than modifiable factors, since they found that dose, volume and fractionation had no significant impact on toxicity. However, with a more appropriate choice of volume parameter, based on the accumulated dose distribution, their conclusions may have been different; they only consider whether the patients have received elective node irradiation or not, rather than evaluating the actual overlapping volumes. In coherence with these results we find no correlation with the size of the PTV at re-irradiation and the toxicity rates. However, when investigating the actual re-treated volume, the overlapping volume, this was found to increase the rate of acute toxicity. This supports the hypothesis that the overlapping volume is a more appropriate volume to evaluate than the PTV in the re-irradiation setting. We are currently exploring the acute and late side effects in re-irradiation for HNC in more detail and investigate the relation to cumulative doses and different organs at risk. The results will be the subject of an accompanying publication.

Several studies have shown that the size of the target volumes has an impact on the outcome at re-irradiation. In a retrospective study by Takiar et al., 227 HNC patients treated with re-irradiation were reviewed and they found that a re-treatment volume (clinical target volume at re-irradiation) $>50 \mathrm{~cm}^{3}$ was associated with increased grade $\geq 3$ toxicity [23]. In the study of Lee et al., based on $66 \mathrm{HNC}$ patients re-irradiated with IMRT, smaller re-treatment volumes (PTV $\leq 100 \mathrm{~cm}^{3}$ ) had significantly reduced risk of severe late toxicity at 2 years (13\% vs. 36\%) [19]. Phan et al. conducted a study of re-irradiation with proton therapy of $60 \mathrm{HNC}$ patients and this study showed that there was a significant association between a clinical target volume $\geq 50 \mathrm{~cm}^{3}$ and acute as well as late grade $\geq 3$ toxicities [29]. A limitation of these studies is that they focus on the impact of target volumes at reirradiation. In the current study, on the other hand, we hypothesized that a larger overlapping volume would have negative effect on patient outcome, such as OS and side effects, supported by the finding that IMRT is associated with a better OS compared to 3D-conformal radiotherapy [16, 17]. However, while in the current study a larger overlapping volume was associated with more acute toxicity it did not result in a lower OS or PFS. The failure to demonstrate such a relationship might be due to the moderate size of the dataset and/or the less specific nature of the latter endpoints. On the one hand, a large overlapping volume could lead to a higher risk of toxicity, but on the other hand, a large overlapping volume could also imply a large tumour volume, which in itself is associated with a poorer prognosis. In the former case, OS and PS might be limited due to the treatment while in the latter they are limited due to the disease. Another limitation of this study is its retrospective nature; despite careful scrutiny of the patient notes relevant information may not have been considered in the analysis. As previously referred to, there are also uncertainties in the accumulated dose distribution.

Nevertheless, this study and its definition of the overlapping re-treated volume could state an example on how re-irradiation and re-irradiation volumes should be defined in future studies. A clear definition on reirradiation and re-irradiation volumes would make it easier to compare results and draw conclusions from different studies. Ultimately, this would make it easier to interpret the results from large studies and use the acquired knowledge in daily practice, when treating the individual patient. In our clinic we will calculate the overlapping volume for future HNC patients that are eligible for re-irradiation and consider this information valuable in selecting patients for re-irradiation with curative intent. This is important knowledge, because re-irradiation can mean cure for the correctly selected patient on one hand, but severe or even fatal toxicity for the poorly selected patient, on the other. The overlapping volume which can safely be treated is yet to be determined and should be the subject of future studies.

\section{Conclusions}

The definition of re-irradiation and re-irradiation volumes is crucial in future studies in re-irradiation of HNC and a necessity to make results from different studies comparable. It is even possible that previous studies have overestimated the benefits of re-irradiation of HNC due to the vague definition of re-irradiation. Our study presents a definition of re-irradiation volume using the data and tools available in modern radiotherapy planning and suggests that larger overlapping volumes in re-irradiation of HNC results in an increase in severe acute side effects. The fact that we did not find a concomitant increase in late effects with increased overlapping volumes should be looked upon with caution and further evaluated with a longer follow up and in a larger patient material. 


\section{Abbreviations}

HNC: head and neck cancer; V100: volume with a cumulative dose of $\geq 100$ Gy; PFS: progression-free survival; OS: overall survival; IMRT: Intensity modulated radiotherapy; PTV: planning target volume; EQD2: equivalent dose of 2 Gy per fraction; VMAT: volumetric modulated arc therapy; CT: computed tomography; D1cc: dose corresponding to the hottest $1 \mathrm{~cm}^{3}$; RTOG: Radiation Oncology Group; EORTC: European Organization for Research and Treatment of Cancer; ECOG: Eastern Cooperative Oncology Group; PS: Performance status; AJCC: American Joint Committee on Cancer

\section{Acknowledgements}

Not applicable.

\section{Authors' contributions}

$A E, E O, C M, I L$ and $S F$ contributed to the design of the study and the interpretation of the results. $A B$ was also involved in the design and interpretation of the statistical methodology, performed the analysis and wrote the section on statistics. AE, EO and SB contributed to the data collection and IL to the registry design and management. SB calculated the cumulative dose plans for all included patients and BW made reconstructions of dose plans not available electronically. AE compiled and presented the results. The manuscript was written by $\mathrm{AE}, \mathrm{EO}, \mathrm{IL}, \mathrm{CM}$ and $\mathrm{SF}$ and was reviewed and approved by all authors.

\section{Funding}

This study was partly funded by a grant (number 164082) from King Gustaf V Jubilee Fund (Stockholm, Sweden). Open access funding provided by Karolinska Institute.

\section{Availability of data and materials}

Research data are stored in an institutional repository and will be shared upon reasonable request to the corresponding author.

\section{Ethics approval and consent to participate}

The study was approved by the Swedish National Ethical Review Authority. Number of ethics approval: 2017/2498-31. The ethical approval states that no consent to participate is required for this study.

\section{Consent for publication}

Not applicable.

\section{Competing interests}

The authors declare that they have no competing interests.

\section{Author details}

'Department of Oncology, Karolinska University Hospital, Anna Steckséns gata 41, 17176 Stockholm, Solna, Sweden. ${ }^{2}$ Department of Oncology-Pathology, Karolinska Institute, Stockholm, Sweden. ${ }^{3}$ Medical Radiation Physics and Nuclear Medicine, Karolinska University Hospital, Stockholm, Sweden. ${ }^{4}$ Epistat Epidemiology and Statistics Consulting, Uppsala, Sweden

Received: 6 April 2020 Accepted: 28 May 2020

Published online: 08 June 2020

\section{References}

1. Pagh A, Grau C, Overgaard J. Failure pattern and salvage treatment after radical treatment of head and neck cancer. Acta Oncol. 2016;55(5):625-32.

2. Goodwin WJ Jr. Salvage surgery for patients with recurrent squamous cell carcinoma of the upper aerodigestive tract: when do the ends justify the means? Laryngoscope. 2000;110(3 Pt 2 Suppl 93):1-18.

3. Gibson MK, Li Y, Murphy B, Hussain MH, DeConti RC, Ensley J, et al. Randomized phase III evaluation of cisplatin plus fluorouracil versus cisplatin plus paclitaxel in advanced head and neck cancer (E1395): an intergroup trial of the eastern cooperative oncology group. J Clin Oncol. 2005;23(15): 3562-7.

4. Vermorken JB, Mesia R, Rivera F, Remenar E, Kawecki A, Rottey S, et al. Platinum-based chemotherapy plus cetuximab in head and neck cancer. N Engl J Med. 2008;359(11):1116-27.
5. Colevas AD. Chemotherapy options for patients with metastatic or recurrent squamous cell carcinoma of the head and neck. J Clin Oncol. 2006;24(17): 2644-52.

6. Argiris A, Harrington KJ, Tahara M, Schulten J, Chomette P, Ferreira Castro A et al. Evidence-based treatment options in recurrent and/or metastatic squamous cell carcinoma of the head and neck. Front Oncol. 2017:7:72.

7. Burtness B, Harrington KJ, Greil R, Soulieres D, Tahara M, de Castro G Jr, et al. Pembrolizumab alone or with chemotherapy versus cetuximab with chemotherapy for recurrent or metastatic squamous cell carcinoma of the head and neck (KEYNOTE-048): a randomised, open-label, phase 3 study. Lancet. 2019;394(10212):1915-28.

8. Ward MC, Riaz N, Caudell JJ, Dunlap NE, Isrow D, Zakem SJ, et al. Refining patient selection for Reirradiation of head and neck squamous carcinoma in the IMRT era: a multi-institution cohort study by the MIRI collaborative. Int J Radiat Oncol Biol Phys. 2018;100(3):586-94.

9. Curtis KK, Ross HJ, Garrett AL, Jizba TA, Patel AB, Patel SH, et al. Outcomes of patients with loco-regionally recurrent or new primary squamous cell carcinomas of the head and neck treated with curative intent reirradiation at Mayo Clinic. Radiat Oncol. 2016;11:55.

10. Duprez F, Berwouts D, Madani I, Bonte K, Boterberg T, De Gersem W, et al. High-dose reirradiation with intensity-modulated radiotherapy for recurrent head-and-neck cancer: disease control, survival and toxicity. Radiother Oncol. 2014;111(3):388-92.

11. Leong YH, Soon YY, Lee KM, Wong LC, Tham IWK, Ho FCH. Long-term outcomes after reirradiation in nasopharyngeal carcinoma with intensitymodulated radiotherapy: a meta-analysis. Head Neck. 2018;40(3):622-31.

12. Sulman EP, Schwartz DL, Le TT, Ang KK, Morrison WH, Rosenthal DI, et al. IMRT reirradiation of head and neck cancer-disease control and morbidity outcomes. Int J Radiat Oncol Biol Phys. 2009;73(2):399-409.

13. Rühle A, Sprave T, Kalckreuth T, Stoian R, Haehl E, Zamboglou C, et al. The value of moderate dose escalation for re-irradiation of recurrent or second primary head-and-neck cancer. Radiat Oncol. 2020;15(1):81.

14. Lee N, Chan K, Bekelman JE, Zhung J, Mechalakos J, Narayana A, et al. Salvage re-irradiation for recurrent head and neck cancer. Int J Radiat Oncol Biol Phys. 2007;68(3):731-40.

15. Beadle BM, Liao KP, Elting LS, Buchholz TA, Ang KK, Garden AS, et al. Improved survival using intensity-modulated radiation therapy in head and neck cancers: a SEER-Medicare analysis. Cancer. 2014;120(5):702-10.

16. Clavel S, Nguyen DH, Fortin B, Despres P, Khaouam N, Donath D, et al. Simultaneous integrated boost using intensity-modulated radiotherapy compared with conventional radiotherapy in patients treated with concurrent carboplatin and 5-fluorouracil for locally advanced oropharyngeal carcinoma. Int J Radiat Oncol Biol Phys. 2012;82(2):582-9.

17. Chen JL, Huang YS, Kuo SH, Hong RL, Ko JY, Lou PJ, et al. Intensitymodulated radiation therapy achieves better local control compared to three-dimensional conformal radiation therapy for T4-stage nasopharyngeal carcinoma. Oncotarget. 2017:8(8):14068-77.

18. Caudell JJ, Ward MC, Riaz N, Zakem SJ, Awan MJ, Dunlap NE, et al. Volume, dose, and fractionation considerations for IMRT-based Reirradiation in head and neck Cancer: a multi-institution analysis. Int J Radiat Oncol Biol Phys. 2018;100(3):606-17.

19. Lee JY, Suresh K, Nguyen R, Sapir E, Dow JS, Arnould GS, et al. Predictors of severe long-term toxicity after re-irradiation for head and neck cancer. Oral Oncol. 2016;60:32-40.

20. Ruifrok AC, van der Kogel AJ. A "reappraisal" of the LQ model for the understanding of dose-fractionation in radiotherapy. Int J Radiat Oncol Biol Phys. 1993;25(5):926-9.

21. Senthi S, Griffioen GH, van Sornsen de Koste JR, Slotman BJ, Senan S. Comparing rigid and deformable dose registration for high dose thoracic re-irradiation. Radiother Oncol. 2013;106(3):323-6.

22. Loi G, Fusella M, Lanzi E, Cagni E, Garibaldi C, lacoviello G, et al. Performance of commercially available deformable image registration platforms for contour propagation using patient-based computational phantoms: a multi-institutional study. Med Phys. 2018;45(2):748-57.

23. Takiar V, Garden AS, Ma D, Morrison WH, Edson M, Zafereo ME, et al. Reirradiation of head and neck cancers with intensity modulated radiation therapy: outcomes and analyses. Int J Radiat Oncol Biol Phys. 2016;95(4):1117-31.

24. Choe KS, Haraf DJ, Solanki A, Cohen EE, Seiwert TY, Stenson KM, et al. Prior chemoradiotherapy adversely impacts outcomes of recurrent and second primary head and neck cancer treated with concurrent chemotherapy and reirradiation. Cancer. 2011;117(20):4671-8. 
25. Dionisi F, Fiorica F, D'Angelo E, Maddalo M, Giacomelli I, Tornari E, et al. Organs at risk's tolerance and dose limits for head and neck cancer reirradiation: a literature review. Oral Oncol. 2019;98:35-47.

26. Spencer SA, Harris J, Wheeler RH, Machtay M, Schultz C, Spanos W, et al. Final report of RTOG 9610, a multi-institutional trial of reirradiation and chemotherapy for unresectable recurrent squamous cell carcinoma of the head and neck. Head Neck. 2008;30(3):281-8.

27. Tanvetyanon T, Padhya T, McCaffrey J, Zhu W, Boulware D, Deconti R, et al. Prognostic factors for survival after salvage reirradiation of head and neck cancer. J Clin Oncol. 2009;27(12):1983-91.

28. Ward MC, Lee NY, Caudell JJ, Zajichek A, Awan MJ, Koyfman SA, et al. A competing risk nomogram to predict severe late toxicity after modern reirradiation for squamous carcinoma of the head and neck. Oral Oncol. 2019; 90:80-6.

29. Phan J, Sio TT, Nguyen TP, Takiar V, Gunn GB, Garden AS, et al. Reirradiation of head and neck cancers with proton therapy: outcomes and analyses. Int J Radiat Oncol Biol Phys. 2016;96(1):30-41.

\section{Publisher's Note}

Springer Nature remains neutral with regard to jurisdictional claims in published maps and institutional affiliations.

Ready to submit your research? Choose BMC and benefit from:

- fast, convenient online submission

- thorough peer review by experienced researchers in your field

- rapid publication on acceptance

- support for research data, including large and complex data types

- gold Open Access which fosters wider collaboration and increased citations

- maximum visibility for your research: over $100 \mathrm{M}$ website views per year

At $\mathrm{BMC}$, research is always in progress.

Learn more biomedcentral.com/submissions 